\title{
Projection Matrix Design for Co-Sparse Analysis Model Based Compressive Sensing
}

\author{
Endra Oey ${ }^{1, *}$, Dadang Gunawan ${ }^{1}$, and Dodi Sudiana ${ }^{1}$ \\ ${ }^{1}$ Wireless and Signal Processing (WASP), Department of Electrical Engineering, University of \\ Indonesia, Depok City, West Java 16424, Indonesia
}

\begin{abstract}
Co-sparse analysis model based-compressive sensing (CAMB$\mathrm{CS}$ ) has gained attention in recent years as alternative to conventional sparse synthesis model based (SSMB)-CS. The equivalent operator as counterpart of the equivalent dictionary in the SSMB-CS is introduced in the CAMB-CS as the product of projection matrix and transpose of the analysis dictionary. This paper proposes an algorithm for designing suitable projection matrix for CAMB-CS by minimizing the mutual coherence of the equivalent operator based on equiangular tight frames design. The simulation results show that the CAMB-CS with the proposed projection matrix outperforms the SSMB-CS in terms of the signal quality reconstruction.
\end{abstract}

\section{Introduction}

Compressive sensing (CS) as a new paradigm in signal acquisition has gained popularity over the last decade after it was introduced in [1-2]. CS acquires the signal directly in already compressed form by projecting it into a well-designed projection matrix. CS framework has been applied in many applications such as imaging applications, internet of thing, data security, and more [3-4]. A conventional CS systems works based on the sparse synthesis model of signal where a signal can be synthesized from a few atoms of a synthesis dictionary [5]. The alternative model is co-sparse model where sparse analysis coefficients can be obtained by multiplying the signal and an analysis dictionary (operator) [6]. Co-sparse analysis model based (CAMB)-CS has attracted attention in recent years because it outperforms the synthesis model as shown in [7-8].

Three main problems of CS are how to build a dictionary, design a proper projection matrix and reconstruct the signal from CS. The famous KSVD algorithm and its extensions have been commonly used to build a synthesis dictionary [9-10] also the improvements by exploiting additional structure of sparse coefficients can be found in [11-12]. The analysis version of KSVD [13] and sparsifying transforms learning algorithms have been used to build an operator [14-15]. The Convex and Relaxation, Greedy, and Bayesian algorithms are used for signal reconstruction in synthesis based CS [16] as well as the counterpart algorithms for analysis based CS [6, 17].

\footnotetext{
* Corresponding author: oey.endra51@ui.ac.id
} 
While how to design optimal projection matrix for sparse synthesis model based (SSMB)-CS has been widely proposed such as in [18-19] but for CAMB-CS has not received attention. This paper addresses how to design a projection matrix for CAMB-CS, use it to perform CS on a natural image and compare the image reconstruction performance to SSMB-CS.

\section{SSMB-CS And CAMB-CS}

In the SSMB-CS, the signal $x \in \mathfrak{R}^{N \times 1}$ is synthesized from a sparse linear combinations of the dictionary columns $\Psi \underline{\underline{\Delta}}\left[\psi_{1} \psi_{2} \ldots \psi_{L}\right] \in \mathfrak{R}^{N \times L}$ :

$$
x=\sum_{i=1}^{L} \theta_{i} \psi_{i} \triangleq \Psi \theta
$$

if $L>N \Psi$ is called overcomplete synthesis dictionary, $\theta \in \mathfrak{R}^{N \times 1}$ is sparse coefficients with $\|\theta\|_{0}=K, K<N$ and $\|\theta\|_{0}$ is number of non-zero elements in $\theta$. The CS is performed by multiplying the signal $x$ and the projection matrix $\Phi \in \mathfrak{R}^{M \times N}$ then $y=\Phi x$, where $y \in \mathfrak{R}^{M \times 1}$ is a compressive measurement vector and $M<N$. The reconstructed signal $\hat{x}$ can be obtained from $y$ by solving the following constrained problem :

$$
\min \|\hat{\theta}\|_{0} \text { s.t. } y=\Phi \Psi \hat{\theta} \text { and } \hat{x}=\Psi \hat{\theta}
$$

The problem in (2) is NP-hard and has combinatorial complexity but can be approximately solved using the Convex and Relaxation, Greedy, or Bayesian algorithms [16].

In CAMB-CS, the analysis coefficients $\alpha \in \mathfrak{R}^{R \times 1}$ are obtained by multiplying the operator $\Omega \in \mathfrak{R}^{R \times N}$ and the signal $x \in \mathfrak{R}^{N \times 1}$ then $\alpha=\Omega x$. If $R>N, \Omega$ is called overcomplete operator. The co-support $\Lambda \subseteq\{1 \ldots R\}$ of $x$ is the index rows of $\Omega$ that are orthogonal to it, so $\Omega_{\Lambda} x=0$ where $|\Lambda|=c$ is co-sparsity of the signal $x$ and $\|\alpha\|_{0}=R-c$ is number of non-zero elements in $\alpha$. The reconstructed signal $\hat{x}$ can be obtained from CAMB-CS by solving the following constrained problem :

$$
\min \|\Omega \hat{x}\|_{0} \text { s.t. } y=\Phi \hat{x}
$$

The problem in (3) can be solved by using the SSMB-CS counterpart algorithms for CAMB-CS [17].

\section{Projection Matrix Design}

In the SSMB-CS, the equivalent dictionary $D \in \Re^{M \times L}$ is defined as $D=\Phi \Psi$ where $G_{s}=D^{T} D$ is the Gram matrix of $D, g_{s-i j}$ is the $(i, j)$ th elements of $G_{s}$ and $Z_{s} \stackrel{\Delta}{=} \operatorname{diag}\left(g_{s-11}^{-1 / 2} \ldots g_{s-l l}^{-1 / 2} \ldots g_{s-L L}^{-1 / 2}\right)$. The normalized equivalent dictionary is $\bar{D} \underline{\underline{\Delta}} D Z_{s}$ and the normalized Gram matrix is $\bar{G}_{s}=\bar{D}^{T} \bar{D}$ such that $\bar{g}_{s-l l}=1, \forall l$. The mutual coherence 
of $D$ is defined as $\mu(D)=\max _{i \neq j}\left|\bar{g}_{s-i j}\right|$ where $\mu(D)$ is bounded with $\mu_{B} \leq \mu(D) \leq 1$ and $\mu_{B} \triangleq \sqrt{\frac{L-M}{M(L-1)}}$ is Welch bound [20]. The $t$-averaged mutual coherence $\mu_{t}(D)$ was introduced in [18] as $\mu_{t}(D):=\frac{\sum_{i \neq j, 1 \leq i, j \leq L}\left(\left|\bar{g}_{s-i j}\right| \geq t\right)\left|\bar{g}_{s-i j}\right|}{\sum_{i \neq j, 1 \leq i, j \leq L}\left(\left|\bar{g}_{s-i j}\right| \geq t\right)}$ where $0 \leq t<1$ and indicator function $\left(\left|\bar{g}_{s-i j}\right| \geq t\right)=1$ if the condition is true and otherwise is zero. The common projection matrix design for SSMB-CS is based on how to make $\mu_{t}(D)$ as small as possible. It is done by making $G$ as close as possible to a target Gram matrix $G_{t}$ that has desired properties such as ETF Gram matrix [20]. The projection matrix design is performed by solving:

$$
\min _{\Phi, G_{t}}\left\|G_{t}-\Psi^{T} \Phi^{T} \Phi \Psi\right\|_{F}^{2}
$$

where \|\|$_{F}$ denotes the Frobenius norm and it can be solved based on shrinkage method [18] or alternating projection [19]. The equivalent operator $O \in \mathfrak{R}^{M \times R}$ is introduced for CAMB-CS as $O=\Phi \Omega^{T}$ where $G_{c}=O^{T} O$ is the Gram matrix of $O, g_{c-i j}$ is the $(i, j)$ th elements of $G_{c}$ and $Z_{c} \triangleq \underline{\Delta} \operatorname{diag}\left(g_{c-11}^{-1 / 2} \ldots g_{c-l l}^{-1 / 2} \ldots g_{c-L L}^{-1 / 2}\right)$. The normalized equivalent operator is $\bar{O} \triangleq O Z_{c}$ and the normalized Gram matrix is $\bar{G}_{c}=\bar{O}^{T} \bar{O}$ such that $\bar{g}_{c-l l}=1, \forall l$. The projection matrix design for CAMB-CS is performed by solving:

$$
\min _{\Phi, G_{t}}\left\|G_{t}-\Omega \Phi^{T} \Phi \Omega^{T}\right\|_{F}^{2}
$$

This paper adapted algorithm in [19] to solve (5) by using the following algorithm which is denoted as OGS algorithm.

\section{Initialization:}

\section{OGS Algorithm}

$$
\begin{aligned}
& \Omega \quad \text { - Operator; } \\
& \Phi^{(0)} \quad \text { - Initial Projection Matrix; } \\
& K \quad \text { - Number of Iterations; } \\
& \text { Set } k=1 \text {. }
\end{aligned}
$$

Begin: For $1 \leq k \leq K$, calculate (6) until (12):

$$
\begin{gathered}
O^{(k-1)}=\Phi^{(k-1)} \Omega^{T} \\
\bar{O}^{(k-1)}=O^{(k-1)} Z_{c} \\
\bar{G}_{c}^{(k-1)}=\left(\bar{O}^{(k-1)}\right)^{T}\left(\bar{O}^{(k-1)}\right)
\end{gathered}
$$




$$
\bar{G}_{c-e}^{(k)}(i, j)=\left\{\begin{array}{cl}
1, & i=j \\
\bar{G}_{c}{ }^{(k-1)}(i, j), & \left|\bar{G}_{c}(i, j)\right| \leq \xi \\
\operatorname{sign}\left(\bar{G}_{c}^{(k-1)}(i, j)\right) \xi & \text { otherwise }
\end{array}\right.
$$

eigenvalue decomposition of $\bar{G}_{c-e}^{(k)}$ :

$$
\bar{G}_{c-e}^{(k)}=V_{e}\left[\begin{array}{cc}
\Lambda_{e} & \mathbf{0} \\
\mathbf{0} & \mathbf{0}
\end{array}\right] V_{e}^{T}
$$

where $V_{e}$ is orthonormal matrix and $\Lambda_{e}=\operatorname{diag}\left[\lambda_{1}, \ldots \lambda_{r}, \ldots, \lambda_{R}\right]$ with $\lambda_{r} \geq \lambda_{r+1}, \forall r$

$$
\begin{aligned}
& O_{e t f}^{(k)}=\frac{U}{\sqrt{M}}\left[\sqrt{\left(\sum_{r=1}^{M} \lambda_{r}\right)} I_{M} \quad \mathbf{0}\right] V_{e}^{T} \\
& \Phi^{(k)}=O_{\text {etf }}{ }^{(k)}\left(\Omega^{T}\right)^{+}
\end{aligned}
$$

where $\left(\Omega^{T}\right)^{+}$is pseudo-inverse of $\left(\Omega^{T}\right)$.

Go to Begin with $k \rightarrow k+1$ to continue the iterative procedure.

End: End the algorithm, output $\Phi=\Phi^{(k-1)}$.

\section{Results and Discussion}

This paper used 1000 training-images in LabelMe training data set [21-22] where 20 nonoverlapping $8 \times 8$ patches are taken randomly from each image and each patch is rearranged as a vector of $64 \times 1$. This training patches $P \in \mathfrak{R}^{64 \times 20000}$ were used to build synthesis dictionary $\Psi \in \mathfrak{R}^{64 \times 96}$ by using KSVD algorithm [9] and operator $\Omega \in \mathfrak{R}^{96 \times 64}$ by using the algorithm in [15]. Algorithm in [19] which is denoted with BLH and OGS algorithm were used for SSMB-CS and CAMB-CS projection matrix design respectively. Both algorithms used the same Gaussian random matrix $\Phi^{(0)} \in \mathfrak{R}^{M \times 64}$ as initial projection matrix, number of iterations $K=1000$ and $\xi=\mu_{B}$. The test image $I^{\text {test }}$ was divided into $8 \times 8$ non-overlapping patches where each patch convert to vector $x_{j} \in \mathfrak{R}^{64 \times 1}, j=1,2, \ldots J$ where $J$ is number of patches in the tes image. CS was performed on those patches to obtain $y_{j} \in \mathfrak{R}^{M \times 64}$. The OMP [23] and its counterpart Greedy algorithm GAP [6] were used for SSMB-CS and CAMB-CS respectively to obtain each reconstructed patch $\hat{x}_{j} \in \mathfrak{R}^{64 \times 1}$ and the whole reconstructed patches are arranged to get the reconstructed image $\hat{I}$. The Peak Signal-to-Noise Ratio (PSNR) was used to measure image reconstruction accuracy. It is defined as PSNR $(\mathrm{dB})=10 \log _{10}\left(\frac{W \times H \times 1^{2}}{\sum_{w=1}^{W} \sum_{h=1}^{H}\left(\hat{I}_{w, h}-I_{w, h}^{\text {test }}\right)^{2}}\right)$ where $W$ and $H$ are width and height of image. 
Table 1 shows the reconstruction (in PSNR (dB)) comparison of SSMB-CS and CAMB-CS for several standard test images with Compression Ratio $(\mathrm{CR})=$ Ratio Measurement Number $(\mathrm{RMN})=\frac{M}{N}=31.25 \%$. Figure 1 shows the PSNR versus CR from $18.75 \%$ until $81.25 \%$ for Brick test image.

Table 1. Reconstruction comparison of SSMB-CS and CAMB-CS for CR $=31.25 \%$.

\begin{tabular}{|c|c|c|c|c|}
\hline \multirow{3}{*}{ Projection Matrix } & \multicolumn{4}{|c|}{$\begin{array}{l}\text { Test Images } \\
\text { (Size) }\end{array}$} \\
\hline & $\begin{array}{c}\text { Moon } \\
(1986 x \\
1986)\end{array}$ & $\begin{array}{c}\text { Brick } \\
(768 \times 512)\end{array}$ & $\begin{array}{c}\text { Barbara } \\
(\mathbf{7 2 0} \times 576)\end{array}$ & $\begin{array}{c}\text { Peppers } \\
(512 \times 512)\end{array}$ \\
\hline & \multicolumn{4}{|c|}{ PSNR (dB) } \\
\hline Random SSMB-CS & 27.48 & 20.86 & 22.52 & 25.14 \\
\hline Random CAMB-CS & 31.46 & 23.61 & 23.93 & 28.45 \\
\hline BLH SSMB-CS & 27.22 & 19.72 & 21.71 & 24.03 \\
\hline OGS CAMB-CS & 33.98 & 25.95 & 25.89 & 31.25 \\
\hline
\end{tabular}

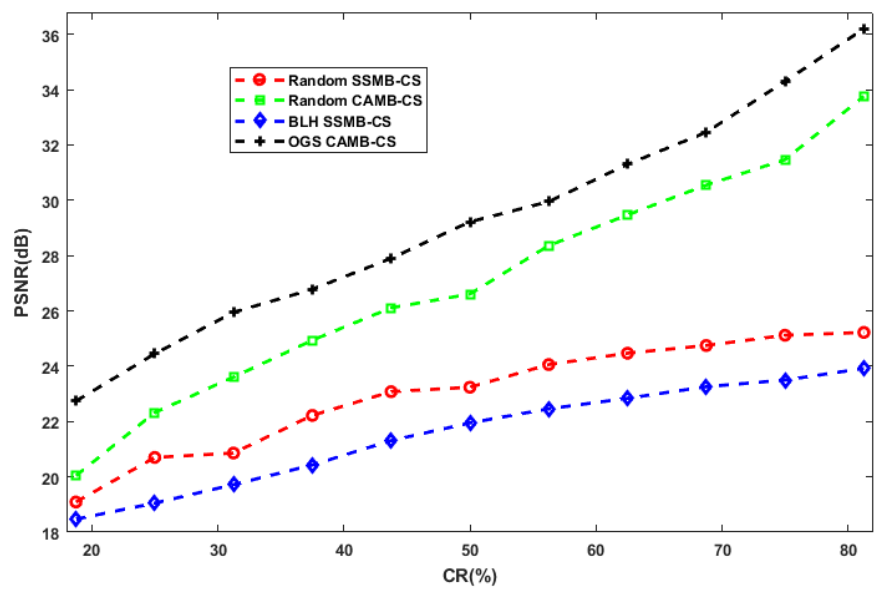

Fig. 1. PSNR (dB) versus CR (\%) of Brick test image for SSMB-CS and CAMB-CS.

From Table 1 and Figure 1, it is clear that the proposed algorithm in this paper (OGS CAMB-CS) outperforms the random projection matrix and the previous algorithm (BLH SSMB-CS). It is noted that the reconstruction time for CAMB-CS is comparable to SSMBCS. Reconstruction time of Barbara test image, as an example, for $\mathrm{CR}=31.25 \%$ are $4.22 \mathrm{~s}$, $6.19 \mathrm{~s}, 4.45 \mathrm{~s}$ and $6.67 \mathrm{~s}$ for Random SSMB-CS, Random CAMB-CS, BLH SSMB-CS and OGS CAMB-CS respectively.

\section{Conclusion}

In this paper, the projection matrix design algorithm for CAMB-CS was proposed to improve image reconstruction accuracy. The results show that CAMB-CS outperforms the SSMB-CS in terms of PSNR of the image reconstruction. Further improvement can be attempted in future work by designing projection matrix and operator learning simultaneously. 


\section{References}

1. D.L. Donoho, Compressed Sensing, IEEE Trans. Inf. Theory 52(4), 1289-1306 (2006).

2. E.J. Candès, J.R, T. Tao, Robust Uncertainty Principles: Exact Signal Reconstruction From Highly Incomplete Frequency Information, IEEE Trans. Inf. Theory 52(2), 489-509 (2006).

3. J.A.M Lorenzo, R. Obermeier, Sensing Matrix Design via Mutual Coherence Minimization for Electromagnetic Compressive Imaging Applications, IEEE Trans. Comp. Img 3(2), 217-229 (2017).

4. S.Qaisar, R.M. Bilal, W. Iqbal, M. Naureen, S. Lee, Compressive Sensing: From Theory to Applications, a Survey, ComNet J 15(5), 443 - 456 (2013).

5. A. M. Bruckstein, D. L. Donoho, M. Elad, From Sparse Solutions of Systems of Equations to Sparse Modeling of Signals and Images, SIAM Rev 51(1), 34-81 (2009).

6. S. Nam, M. Davies, M. Elad, R.Gribonval, The Cosparse Analysis Model and Algorithms, Appl. Comput. Harmon. Anal 34(1), 30-56 (2013).

7. O. Endra, D. Gunawan, Comparison of Synthesis-Based and Analysis-Based Compressive Sensing, In Proceedings of IEEE International Conference QiR, 167-170 (2015).

8. R. Saiprasad, Y. Bresler, Data-Driven Learning of a Union of Sparsifying Transforms Model for Blind Compressed Sensing, IEEE Trans. Comp. Img. 2(3), 294-309 (2016).

9. M. Aharon, M. Elad, A. Bruckstein, The KSVD: An Algorithm for Designing Overcomplete Dictionaries for Sparse Representation, IEEE Trans. Sig. Proc, 54(11), 4311-4322 (2006).

10. I. Kviatkovsky, M. Gabel, E. Rivlin, I. Shimshoni, On the Equivalence of the LC-KSVD and the D-KSVD Algorithms, IEEE Trans. Pattern Analysis and Machine Intelligence, 39(2), 411-416 (2017).

11. M.F. Duarte, V. Cevher, R.G. Baraniuk, Model-based compressive sensing for signal ensembles, In Proceedings of $47^{\text {th }}$ Annual IEEE Allerton Conference on Communication, Control, and Computing, 244-250 (2009).

12. C. Hegde, P. Indyk, L. Schmidt, Approximation-tolerant model-based compressive sensing, In Proceedings of $25^{\text {th }}$ Annual ACM-SIAM Symposium on Discrete Algorithms, 1544-1561 (2014).

13. R. Rubinstein, T. Peleg, M. Elad, Analysis K-SVD: A Dictionary-Learning Algorithm for the Analysis Sparse Model. IEEE Trans. Sig. Proc, 61(3), 661-677 (2013).

14. S. Ravishankar, Y. Bresler, Learning Sparsifying Transforms, IEEE Trans. Sig. Proc 61(5), 1072-1086 (2013).

15. B. Hou, Z. Zhu, G. Li, A. Yu, An Efficient Algorithm for Overcomplete Sparsifying Transform Learning with Signal Denoising, Math. Problems. in Eng. (2016).

16. Y. Arjoune, N. Kaabouch, H.E. Ghazi, A. Tamtaoui, Compressive Sensing: Performance Comparison of Sparse Recovery Algorithms, In IEEE $7^{\text {th }}$ Annual Computing and Communication Workshop and Conference (CCWC), 1-7 (2017).

17. R.Giryes, A Greedy Algorithm for the Analysis Transform Domain, Neurocomputing 173, 278289 (2016).

18. M. Elad, Optimized Projections for Compressed Sensing, IEEE Trans. Sig. Proc, 55(12), 56955702 (2007).

19. H. Bai, S. Li, X. He, Sensing Matrix Optimization Based on Equiangular Tight Frames with Consideration of Sparse Representation Error, IEEE Trans. Multimedia, 18(10), 2040-2053 (2016).

20. T. Strohmer, R.W. Heath, Grassmannian Frames with Applications to Coding and Communication, Appl. Comp. Harmon. Analysis, 14(3) (2003).

21. B. C. Russell, A. Torralba, K. P. Murphy, W. T. Freeman, LabelMe: A Database and Web-Based Tool for Image Annotation, Comput. Vis. J. 77(1), 157-173 (2008).

22. R. Uetz, S. Behnke, Large-Scale Object Recognition with CUDA-Accelerated Hierarchical Neural Networks, In Proceedings of IEEE ICIS, 536-541 (2009).

23. J. A. Tropp, A.C. Gilbert, Signal Recovery from Random Measurements via Orthogonal Matching Pursuit, IEEE Trans. Inf. Theory 53(12), 4655-4666 (2007). 